\title{
Genetic pathways of multiple esophageal squamous cell carcinomas
}

\author{
TAKAYASU KUWABARA ${ }^{1}$, TORU HIYAMA ${ }^{4}$, SHINJI TANAKA ${ }^{2}$, MASAHARU YOSHIHARA $^{4}$, \\ KOJI ARIHIRO $^{3}$ and KAZUAKI CHAYAMA ${ }^{1}$ \\ Departments of ${ }^{1}$ Gastroenterology and Metabolism, ${ }^{2}$ Endoscopy and ${ }^{3}$ Anatomical Pathology, Hiroshima University \\ Hospital, 1-2-3 Kasumi, Minami-ku, Hiroshima 734-8551; ${ }^{4}$ Health Service Center, Hiroshima University, \\ 1-7-1 Kagamiyama, Higashihiroshima 739-8514, Japan
}

Received September 28, 2010; Accepted November 10, 2010

DOI: $10.3892 /$ or.2010.1110

\begin{abstract}
Whether multiple esophageal squamous cell carcinomas (SCCs) in a patient develop through an identical genetic pathway is still unclear. We examined multiple esophageal SCCs for alterations of $p 53, p 16, I R F$ and mitochondrial DNA (mtDNA) and microsatellite instability (MSI). Thirty patients with multiple superficial esophageal SCCs, 23 with double lesions and 7 with triple lesions, were enrolled. Loss of heterozygosity (LOH) of p53 (TP53), p16 (D9S171), IRF (IRF) and other microsatellite loci including D1S191, D17S858, D18S58 and D18S61 of the tumors was examined by microsatellite assay. Mutations of p16 and mtDNA were examined with PCR single-strand conformation polymorphism (SSCP) analysis. LOH of p53, p16 and IRF were detected in 16 of $50(32 \%), 5$ of $38(13 \%)$ and 5 of $48(10 \%)$ tumors, respectively. Mutations of p16 were detected in 4 of $67(6 \%)$ tumors. Six of $67(9 \%)$ tumors had mtDNA alterations and none of the tumors showed high-frequency MSI. All 30 patients showed one or more gene alterations in one or more genetic loci. Discordant genetic patterns among individual lesions within a patient were observed in 28 of the 30 (93\%) patients. The most discordant locus was TP53, present in 11 of $29(38 \%)$ informative cases, followed by D18S61, present in 11 of $30(37 \%)$ informative cases. These results suggest that the genetic pathways of multiple esophageal SCCs may differ even within the same patient.
\end{abstract}

\section{Introduction}

Multiple esophageal squamous cell carcinomas (SCCs) are observed in $20-30 \%$ of patients with esophageal SCC $(1,2)$.

Correspondence to: Dr Toru Hiyama, Health Service Center, Hiroshima University, 1-7-1 Kagamiyama, Higashihiroshima 7398514, Japan

E-mail: tohiyama@hiroshima-u.ac.jp

Key words: multiple esophageal squamous cell carcinomas, $p 53$, $p 16, I R F$, mitochondrial DNA
We previously reported that the mean annual incidence of newly diagnosed tumors was $4.4 \%$ in patients with esophageal SCC (1). Interestingly, patients with synchronous esophageal SCCs were reported to have a significantly worse prognosis than those with metachronous esophageal SCCs because patients with metachronous SCCs have a higher rate of earlystage SCC than do those with synchronous SCCs (3). The number of patients with esophageal SCCs has increased because both the Lugol spraying method and narrow-band imaging facilitate the early detection of this carcinoma (1).

There are two theories regarding multiple carcinogenesis in an organ. One is the multicentric carcinogenesis theory, which postulates that cells from a single neoplastic cell may be developed in multiple cites independently (4). The other is the field carcinogenesis theory, which postulates that an area of tissue simultaneously becomes genetically unstable and is predisposed to neoplasia due to prolonged exposure to carcinogens, resulting in multiple tumors (5). Esophageal SCC is regarded as a representative example of field carcinogenesis (6). According to the theory, the entire esophageal epithelial surface or 'field' is exposed to repeated carcinogenic insults, and multiple epithelial tumors can arise from multifocal precancerous lesions, which may develop and progress at different rates. Whether multiple esophageal SCCs within a patient develop through an identical genetic pathway is of great interest. Although multiple genetic alterations, such as mutations of $p 53, p 16$, interferon-regulatory factor $(I R F)$ and mitochondrial DNA (mtDNA), and microsatellite instability (MSI) are reported to be involved in the development of esophageal SCC (7-9), little information is available regarding the molecular alterations of multiple esophageal SCCs. Therefore, we examined multiple esophageal SCCs for alterations of $p 53, p 16, I R F$ and mtDNA, and MSI to evaluate this issue.

\section{Patients and methods}

Patients. Thirty Japanese patients with multiple superficial esophageal SCCs, 23 with double cancers and 7 with triple cancers, who had been treated endoscopically at Hiroshima University Hospital from November 1994 to September 2006 without radiation or chemotherapy prior to the treatment, were enrolled in this study. Superficial esophageal SCC is 
Table I. Characteristics of patients with multiple esophageal SCCs.

\begin{tabular}{|c|c|c|c|c|c|c|}
\hline & Age (years) & Gender & Location ${ }^{a}$ & Morphologic type $^{b}$ & Size (mm) & Depth $^{c}$ \\
\hline $1-\mathrm{A}$ & 75 & M & $\mathrm{Lt}$ & 0 -IIc & 9 & EP \\
\hline $1-B$ & 78 & M & $\mathrm{Lt}$ & 0-IIc & 20 & EP \\
\hline $2-A$ & 68 & M & Mt & 0 -IIc & 8 & EP \\
\hline $2-B$ & 68 & M & Mt & 0-IIc & 10 & EP \\
\hline $3-A$ & 64 & M & Mt & 0-IIc & 25 & EP \\
\hline $3-B$ & 64 & M & $\mathrm{Lt}$ & 0 -IIc & 10 & EP \\
\hline $4-\mathrm{A}$ & 68 & M & Mt & 0-IIc & 45 & EP \\
\hline $4-B$ & 69 & M & $\mathrm{Lt}$ & 0 -IIc & 5 & EP \\
\hline $5-\mathrm{A}$ & 63 & M & Mt & 0-IIc & 15 & EP \\
\hline $5-\mathrm{B}$ & 63 & M & $\mathrm{Lt}$ & 0-IIc & 10 & EP \\
\hline $6-\mathrm{A}$ & 76 & M & Mt & 0-IIc & 60 & LPM \\
\hline $6-B$ & 76 & M & Mt & 0-IIc & 15 & EP \\
\hline $6-C$ & 76 & M & Mt & 0 -IIc & 5 & EP \\
\hline 7-A & 67 & M & Mt & 0-IIc & 25 & LPM \\
\hline 7-B & 67 & $\mathrm{M}$ & Mt & 0 -IIc & 20 & EP \\
\hline $8-\mathrm{A}$ & 72 & M & $\mathrm{Lt}$ & 0-IIc & 60 & MM \\
\hline $8-\mathrm{B}$ & 76 & M & $\mathrm{Lt}$ & 0-IIc & 30 & MM \\
\hline $9-\mathrm{A}$ & 69 & M & $\mathrm{Lt}$ & 0-IIc & 10 & LPM \\
\hline $9-\mathrm{B}$ & 70 & M & $\mathrm{Lt}$ & 0-IIc & 8 & EP \\
\hline $10-\mathrm{A}$ & 67 & $\mathrm{M}$ & $\mathrm{Lt}$ & 0 -IIc & 30 & SM1 \\
\hline $10-B$ & 74 & $\mathrm{M}$ & $\mathrm{Ce}$ & 0-IIc & 50 & EP \\
\hline $11-\mathrm{A}$ & 50 & $\mathrm{M}$ & $\mathrm{Lt}$ & 0-IIc & 15 & LPM \\
\hline 11-B & 51 & $\mathrm{M}$ & $\mathrm{Lt}$ & 0-IIc & 25 & LPM \\
\hline $12-\mathrm{A}$ & 55 & $\mathrm{M}$ & $\mathrm{Lt}$ & 0-IIc & 10 & EP \\
\hline $12-\mathrm{B}$ & 56 & $\mathrm{M}$ & Ut & 0-IIc & 5 & EP \\
\hline $12-\mathrm{C}$ & 56 & $\mathrm{M}$ & $\mathrm{Lt}$ & 0-IIc & 8 & EP \\
\hline $13-\mathrm{A}$ & 56 & $\mathrm{M}$ & $\mathrm{Lt}$ & 0-IIc & 10 & LPM \\
\hline 13-B & 57 & $\mathrm{M}$ & Ut & 0-IIc & 7 & EP \\
\hline $14-\mathrm{A}$ & 78 & $\mathrm{M}$ & Ut & 0-IIc & 10 & LPM \\
\hline $14-B$ & 79 & M & $\mathrm{Lt}$ & 0 -IIc & 12 & LPM \\
\hline $15-\mathrm{A}$ & 57 & $\mathrm{M}$ & Ut & 0-IIc & 25 & $\mathrm{MM}$ \\
\hline $15-\mathrm{B}$ & 61 & $\mathrm{M}$ & Ut & 0-IIc & 25 & EP \\
\hline $16-\mathrm{A}$ & 74 & $\mathrm{~F}$ & Ut & 0 -IIc & 20 & MM \\
\hline $16-\mathrm{B}$ & 74 & $\mathrm{~F}$ & Ut & 0-IIc & 15 & LPM \\
\hline $17-\mathrm{A}$ & 54 & $\mathrm{M}$ & Mt & 0 -IIc & 15 & MM \\
\hline $17-B$ & 54 & $\mathrm{M}$ & $\mathrm{Lt}$ & 0-IIc & 25 & EP \\
\hline $17-\mathrm{C}$ & 56 & $\mathrm{M}$ & Ut & 0-IIc & 20 & EP \\
\hline $18-\mathrm{A}$ & 71 & $\mathrm{M}$ & $\mathrm{Lt}$ & 0 -IIc & 8 & MM \\
\hline $18-\mathrm{B}$ & 74 & $\mathrm{M}$ & $\mathrm{Lt}$ & 0-IIa & 20 & SM2 \\
\hline $19-\mathrm{A}$ & 75 & $\mathrm{M}$ & $\mathrm{Lt}$ & 0 -IIc & 10 & EP \\
\hline $19-\mathrm{B}$ & 75 & $\mathrm{M}$ & $\mathrm{Lt}$ & 0 -IIc & 10 & EP \\
\hline $19-\mathrm{C}$ & 75 & $\mathrm{M}$ & $\mathrm{Lt}$ & 0-IIc & 10 & EP \\
\hline $20-\mathrm{A}$ & 80 & $\mathrm{M}$ & Mt & 0-IIc & 140 & $\mathrm{MM}$ \\
\hline $20-B$ & 80 & $\mathrm{M}$ & Mt & 0 -IIc & 30 & SM2 \\
\hline $21-\mathrm{A}$ & 76 & M & Mt & 0-IIc & 50 & $\mathrm{MM}$ \\
\hline 21-B & 78 & M & $\mathrm{Lt}$ & 0-IIc & 20 & EP \\
\hline $22-\mathrm{A}$ & 54 & $\mathrm{M}$ & Mt & 0-IIc & 20 & $\mathrm{MM}$ \\
\hline $22-B$ & 54 & M & Mt & 0-IIc & 15 & EP \\
\hline $23-A$ & 50 & M & Mt & 0-IIc & 40 & EP \\
\hline $23-\mathrm{B}$ & 50 & M & $\mathrm{Lt}$ & 0-IIc & 15 & EP \\
\hline
\end{tabular}


Table I. Continued.

\begin{tabular}{|c|c|c|c|c|c|c|}
\hline & Age (years) & Gender & Location $^{\mathrm{a}}$ & Morphologic type ${ }^{b}$ & Size $(\mathrm{mm})$ & Depth $^{c}$ \\
\hline 24-A & 62 & M & $\mathrm{Ut}$ & 0-IIc & 20 & EP \\
\hline 24-B & 62 & M & $\mathrm{Lt}$ & 0-IIc & 50 & MM \\
\hline $25-A$ & 63 & M & Mt & 0 -IIc & 30 & SM2 \\
\hline $25-B$ & 63 & M & $\mathrm{Lt}$ & 0-IIc & 40 & EP \\
\hline $26-A$ & 60 & M & $\mathrm{Lt}$ & 0-IIc & 10 & MM \\
\hline 26-B & 60 & M & $\mathrm{Lt}$ & 0-IIc & 15 & LPM \\
\hline 27-A & 66 & M & Mt & 0-IIc & 10 & MM \\
\hline 27-B & 66 & M & Mt & 0-IIc & 10 & LPM \\
\hline $27-\mathrm{C}$ & 66 & M & Mt & 0-IIc & 30 & MM \\
\hline $28-\mathrm{A}$ & 68 & M & Mt & 0-IIc & 10 & EP \\
\hline $28-\mathrm{B}$ & 69 & M & Mt & 0-IIc & 5 & EP \\
\hline $28-\mathrm{C}$ & 69 & M & Mt & 0-IIc & 10 & EP \\
\hline 29-A & 74 & M & Mt & 0-IIc & 20 & MM \\
\hline 29-B & 74 & M & $\mathrm{Lt}$ & 0-IIc & 12 & MM \\
\hline $29-\mathrm{C}$ & 74 & $\mathrm{M}$ & $\mathrm{Ut}$ & 0-IIc & 10 & LPM \\
\hline $30-A$ & 58 & M & Mt & 0-IIc & 25 & MM \\
\hline 30-B & 58 & M & $\mathrm{Lt}$ & 0-IIc & 20 & LPM \\
\hline
\end{tabular}

${ }^{\mathrm{a}} \mathrm{Ce}$, cervical esophagus; Lt, lower thoracic esophagus; Mt, middle thoracic esophagus; Ut, upper thoracic esophagus. ${ }^{\mathrm{b}} 0$-IIa, slightly elevated type; 0-IIc, slightly depressed type. ${ }^{~} \mathrm{EP}$, epithelium; LPM, lamina propria mucosae; MM, muscularis mucosae; SM, submucosal layer. SCC, squamous cell carcinoma.

defined as a lesion that is confined to the esophageal mucosa or submucosa, regardless of the presence or absence of regional lymph node metastasis (10). The description of the esophageal SCCs and histologic evaluation of the resected specimens were in accordance with the Japanese Classification of Esophageal Carcinoma (10). Tumor locations were defined as the cervical esophagus (Ce), upper thoracic esophagus (Ut), middle thoracic esophagus (Mt), lower thoracic esophagus (Lt) and the abdominal esophagus (Ae). The depth of invasion of superficial esophageal SCC was classified as intramucosal or submucosal. The depth of intramucosal invasion was subclassified as epithelium (EP), lamina propria mucosae (LPM) and muscularis mucosae (MM), and the depth of submucosal invasion was subclassified as SM1 to SM3 according to the grade of invasion (1, mild; 2, moderate; or 3, remarkable).

Clinicopathologic features of the 30 patients are shown in Table I. Mean age of the patients was $66.3 \pm 8.6$ years (range, 50-80 years) and male/female ratio was 29/1. Morphologically, of the 67 tumors, 66 were classified as O-IIc or slightly depressed type and 1 was classified as O-IIa or slightly elevated type. Tumor depth was EP in 34 tumors, LPM in 13, MM in 16, SM2 in 3 and SM1 in 1.

The following criteria were used for the diagnosis of primary multiple esophageal SCCs: i) each cancerous lesion histologically showed definite malignant features and was located individually with no continuity and ii) concomitant carcinomas accompanied the areas of intraepithelial carcinomas. The purpose of the latter criterion was to exclude intramural metastatic lesions, which frequently occur in patients with esophageal SCC (6). Lesions detected within 1 year of the initial endoscopic treatment were regarded as synchronous multiple lesions because the presence of microcarcinoma might have been missed at the time of endoscopic treatment. Metachronous multiple lesions were defined as new esophageal SCCs occurring in different areas from the initial cancer and at least 1 year after the initial endoscopic treatment (1).

DNA extraction. Tissue sections $(10-\mu \mathrm{m}$ thick) were stained with hematoxylin and eosin, dehydrated in a graded ethanol series, and then dried without a cover glass. Tissue samples from tumors and corresponding normal tissues were cut with sterile needles, and the DNA was extracted with $20 \mu 1$ of extraction buffer (100 mM Tris- $\mathrm{HCl}, \mathrm{pH} 8.0,2$ mM EDTA, $400 \mu \mathrm{g} / \mathrm{ml}$ proteinase $\mathrm{K}$ ) at $55^{\circ} \mathrm{C}$ overnight. The tubes were boiled for $5 \mathrm{~min}$ to inactivate proteinase $\mathrm{K}$, and 1-2 $\mu \mathrm{l}$ of each extract was used for polymerase chain reaction (PCR) amplification.

Loss of heterozygosity analysis of p53, p16, IRF and other microsatellite loci. Loss of heterozygosity $(\mathrm{LOH})$ analysis of p53 (TP53), p16 (D9S171), IRF (IRF) and other microsatellite loci including D1S191, D17S858, D18S58 and D18S61 was performed with microsatellite assay as described previously (11). In brief, each $15-\mu 1$ reaction mixture contained $10-20 \mathrm{ng}$ genomic DNA, 6.7 mM Tris-HCl, $\mathrm{pH} 8.8,6.7 \mathrm{mM}$ EDTA, $6.7 \mathrm{mM} \mathrm{MgCl}{ }_{2}, 0.175 \mu \mathrm{M}$ primer set, $1.5 \mathrm{mM}\left[\alpha^{32}-\mathrm{P}\right]-\mathrm{dCTP}$, 
Table II. Results of genetic analysis in each patient with multiple esophageal SCCs.

\begin{tabular}{|c|c|c|c|c|c|c|c|c|c|c|}
\hline & p53 (ТP53) & p53 (D9S171) & $p 16$ (Ex 1-3) & $\operatorname{IRF}(\mathrm{IRF})$ & D1S191 & D17S858 & D18S58 & D18S61 & BAT26 & mtDNA \\
\hline $1-\mathrm{A}$ & - & Hom & - & Hom & MSI & Hom & - & - & - & - \\
\hline $1-B$ & - & Hom & Mut (Ex 3) & MSI & $\mathrm{LOH}$ & Hom & - & - & - & - \\
\hline $2-\mathrm{A}$ & - & Hom & - & $\mathrm{LOH}$ & - & Hom & - & Hom & - & - \\
\hline $2-B$ & $\mathrm{LOH}$ & Hom & - & - & - & Hom & - & Hom & - & - \\
\hline $3-\mathrm{A}$ & $\mathrm{LOH}$ & Hom & - & - & - & NI & Hom & - & - & - \\
\hline $3-B$ & - & Hom & - & - & - & NI & Hom & - & - & - \\
\hline $4-\mathrm{A}$ & - & - & - & - & - & Hom & Hom & - & - & - \\
\hline $4-B$ & $\mathrm{LOH}$ & - & - & - & - & Hom & Hom & $\mathrm{LOH}$ & - & - \\
\hline $5-\mathrm{A}$ & - & - & - & - & Hom & Hom & - & - & - & - \\
\hline $5-B$ & - & - & - & - & Hom & Hom & - & - & - & - \\
\hline $6-A$ & - & Hom & - & - & Hom & NI & Hom & - & - & - \\
\hline $6-B$ & $\mathrm{LOH}$ & Hom & - & - & Hom & NI & Hom & - & - & - \\
\hline $6-\mathrm{C}$ & - & Hom & - & - & Hom & NI & Hom & $\mathrm{LOH}$ & - & - \\
\hline $7-A$ & $\mathrm{NI}$ & Hom & - & - & - & Hom & - & - & - & - \\
\hline $7-B$ & NI & Hom & - & $\mathrm{LOH}$ & $\mathrm{LOH}$ & Hom & $\mathrm{LOH}$ & - & - & - \\
\hline $8-A$ & - & Hom & Mut (Ex 2) & - & MSI & Hom & Hom & - & - & - \\
\hline $8-B$ & - & Hom & Mut (Ex 1) & - & Hom & Hom & Hom & - & - & - \\
\hline 9-A & - & - & - & NI & Hom & MSI & Hom & NI & - & - \\
\hline $9-B$ & - & MSI & - & NI & Hom & Hom & Hom & NI & - & - \\
\hline $10-\mathrm{A}$ & $\mathrm{LOH}$ & - & - & $\mathrm{LOH}$ & $\mathrm{LOH}$ & - & Hom & - & - & - \\
\hline $10-B$ & - & - & - & - & - & - & Hom & $\mathrm{LOH}$ & - & - \\
\hline $11-\mathrm{A}$ & - & - & - & MSI & $\mathrm{LOH}$ & Hom & Hom & - & - & - \\
\hline $11-B$ & - & - & - & - & $\mathrm{LOH}$ & Hom & Hom & - & - & - \\
\hline $12-\mathrm{A}$ & $\mathrm{LOH}$ & - & - & - & MSI & - & - & - & - & - \\
\hline $12-B$ & - & - & - & - & Hom & $\mathrm{LOH}$ & - & $\mathrm{LOH}$ & - & - \\
\hline $12-\mathrm{C}$ & - & - & - & - & Hom & - & - & $\mathrm{LOH}$ & - & - \\
\hline $13-\mathrm{A}$ & Hom & - & Mut (Ex 1) & $\mathrm{LOH}$ & MSI & Hom & Hom & - & - & - \\
\hline $13-\mathrm{B}$ & Hom & - & - & NI & - & Hom & Hom & - & - & - \\
\hline $14-\mathrm{A}$ & Hom & Hom & - & NI & - & Hom & Hom & NI & - & - \\
\hline $14-B$ & Hom & Hom & - & NI & - & Hom & Hom & NI & - & - \\
\hline $15-\mathrm{A}$ & Hom & $\mathrm{LOH}$ & - & - & MSI & Hom & Hom & - & - & - \\
\hline $15-\mathrm{B}$ & Hom & - & - & - & Hom & Hom & Hom & - & - & - \\
\hline $16-A$ & - & $\mathrm{LOH}$ & - & - & $\mathrm{LOH}$ & $\mathrm{LOH}$ & MSI & NI & - & Mut \\
\hline $16-B$ & $\mathrm{NI}$ & - & - & MSI & $\mathrm{LOH}$ & $\mathrm{LOH}$ & Hom & NI & - & - \\
\hline $17-\mathrm{A}$ & - & - & - & - & - & Hom & - & - & - & - \\
\hline $17-B$ & - & - & - & - & $\mathrm{LOH}$ & Hom & - & $\mathrm{LOH}$ & - & Mut \\
\hline $17-\mathrm{C}$ & - & $\mathrm{LOH}$ & - & - & $\mathrm{LOH}$ & Hom & $\mathrm{LOH}$ & $\mathrm{LOH}$ & - & - \\
\hline $18-\mathrm{A}$ & NI & Hom & - & - & Hom & NI & - & - & - & - \\
\hline $18-\mathrm{B}$ & $\mathrm{LOH}$ & Hom & - & - & MSI & $\mathrm{LOH}$ & - & - & - & - \\
\hline $19-\mathrm{A}$ & $\mathrm{LOH}$ & $\mathrm{LOH}$ & - & Hom & Hom & $\mathrm{LOH}$ & - & - & - & Mut \\
\hline $19-B$ & - & - & - & Hom & Hom & - & $\mathrm{LOH}$ & - & - & Mut \\
\hline $19-\mathrm{C}$ & - & - & - & Hom & Hom & - & - & $\mathrm{LOH}$ & - & Mut \\
\hline $20-\mathrm{A}$ & $\mathrm{LOH}$ & - & - & Hom & - & - & $\mathrm{LOH}$ & - & - & Mut \\
\hline $20-B$ & $\mathrm{LOH}$ & - & - & Hom & - & - & - & - & - & - \\
\hline $21-\mathrm{A}$ & $\mathrm{LOH}$ & - & - & Hom & - & - & $\mathrm{LOH}$ & - & - & - \\
\hline $21-B$ & $\mathrm{LOH}$ & $\mathrm{LOH}$ & - & Hom & - & - & $\mathrm{LOH}$ & $\mathrm{LOH}$ & - & - \\
\hline $22-\mathrm{A}$ & $\mathrm{LOH}$ & - & - & Hom & Hom & - & - & Hom & - & - \\
\hline $22-B$ & - & - & - & Hom & Hom & - & - & Hom & - & - \\
\hline $23-\mathrm{A}$ & $\mathrm{LOH}$ & Hom & - & - & Hom & - & MSI & - & - & - \\
\hline $23-B$ & - & Hom & - & - & Hom & - & $\mathrm{LOH}$ & - & - & - \\
\hline
\end{tabular}


Table II. Continued.

\begin{tabular}{|c|c|c|c|c|c|c|c|c|c|c|}
\hline & p53 (ТР53) & p53 (D9S171) & $p 16(\operatorname{Ex} 1-3)$ & $\operatorname{IRF}(\mathrm{IRF})$ & D1S191 & D17S858 & D18S58 & D18S61 & BAT26 & mtDNA \\
\hline $24-\mathrm{A}$ & - & Hom & - & Hom & MSI & - & Hom & $\mathrm{LOH}$ & - & - \\
\hline $24-B$ & $\mathrm{LOH}$ & Hom & - & Hom & Hom & - & Hom & - & - & - \\
\hline $25-\mathrm{A}$ & Hom & - & - & Hom & $\mathrm{LOH}$ & - & Hom & - & - & - \\
\hline $25-B$ & Hom & - & - & Hom & - & - & Hom & - & - & - \\
\hline $26-\mathrm{A}$ & MSI & NI & - & - & Hom & NI & NI & $\mathrm{LOH}$ & - & - \\
\hline $26-B$ & - & $\mathrm{NI}$ & - & - & Hom & NI & NI & - & - & - \\
\hline $27-\mathrm{A}$ & Hom & - & - & - & Hom & NI & $\mathrm{NI}$ & - & - & - \\
\hline $27-B$ & Hom & - & - & - & Hom & $\mathrm{NI}$ & NI & $\mathrm{LOH}$ & - & - \\
\hline $27-C$ & Hom & - & - & - & Hom & NI & $\mathrm{LOH}$ & $\mathrm{LOH}$ & - & - \\
\hline $28-\mathrm{A}$ & - & NI & - & - & Hom & $\mathrm{NI}$ & - & Hom & - & - \\
\hline $28-B$ & - & NI & - & - & Hom & NI & $\mathrm{LOH}$ & Hom & - & - \\
\hline $28-\mathrm{C}$ & - & NI & - & - & Hom & NI & - & Hom & - & - \\
\hline $29-\mathrm{A}$ & $\mathrm{LOH}$ & Hom & - & - & Hom & NI & Hom & - & - & - \\
\hline 29-B & NI & Hom & - & - & Hom & $\mathrm{NI}$ & Hom & - & - & - \\
\hline $29-\mathrm{C}$ & NI & Hom & - & MSI & Hom & NI & Hom & - & - & - \\
\hline $30-\mathrm{A}$ & - & $\mathrm{LOH}$ & - & - & Hom & NI & - & Hom & - & - \\
\hline $30-B$ & - & $\mathrm{LOH}$ & - & $\mathrm{LOH}$ & Hom & NI & - & Hom & - & - \\
\hline
\end{tabular}

SCC, squamous cell carcinoma; Hom, homozygous allele; LOH, loss of heterozygosity (+); MSI, microsatellite instability (+); Mut, mutation (+); -, no alteration; NI, not informative.

$1.5 \mathrm{mM}$ of each deoxynucleotide triphosphate and 0.75 units AmpliTaq Gold DNA polymerase (Perkin-Elmer, Branchburg, $\mathrm{NJ})$. The reaction mixtures were heated to $95^{\circ} \mathrm{C}$ for $10 \mathrm{~min}$, followed by 45 cycles of denaturation at $94^{\circ} \mathrm{C}$ for $1 \mathrm{~min}$, annealing at $55^{\circ} \mathrm{C}$ for $2 \mathrm{~min}$ and strand elongation at $72^{\circ} \mathrm{C}$ for $2 \mathrm{~min}$. PCR products were separated by electrophoresis on $6 \%$ polyacrylamide- $8 \mathrm{M}$ urea- $32 \%$ formamide gels and subjected to autoradiography overnight at $-80^{\circ} \mathrm{C}$ on Fuji RX film. LOH was identified when only one major band was detected in DNA isolated from cancerous tissue; two major bands were present in the normal tissue specimen from the same sample. MSI was identified when additional bands not present in the normal tissue DNA were detected. Microsatellite assay with BAT26 was used to evaluate high-frequency MSI (MSI-H) (12).

Mutational analysis of p16 and mtDNA. Mutations of $p 16$ and the D310 region of mtDNA were examined with PCRsingle-strand conformation polymorphism (SSCP) analysis as described previously (13). In brief, In brief, each $15-\mu 1$ reaction mixture contained 10-20 ng genomic DNA, $6.7 \mathrm{mM}$ Tris- $\mathrm{HCl}$, pH 8.8, $6.7 \mathrm{mM}$ EDTA, $6.7 \mathrm{mM} \mathrm{MgCl}_{2}, 0.175 \mu \mathrm{M}$ primer set, $1.5 \mathrm{mM}\left[\alpha^{32}-\mathrm{P}\right]-\mathrm{dCTP}, 1.5 \mathrm{mM}$ of each deoxynucleotide triphosphate and 0.75 units AmpliTaq Gold DNA polymerase. The reaction mixtures were heated to $95^{\circ} \mathrm{C}$ for $10 \mathrm{~min}$, followed by 45 cycles of denaturation at $94^{\circ} \mathrm{C}$ for $1 \mathrm{~min}$, annealing at $55^{\circ} \mathrm{C}$ for $2 \mathrm{~min}$, and strand elongation at $72^{\circ} \mathrm{C}$ for $2 \mathrm{~min}$. After PCR, the samples were electrophoresed on $6 \%$ polyacrylamide gels (acrylamide:bis-acrylamide ratio, 19:1) with $10 \%$ glycerol at $4^{\circ} \mathrm{C}$. The gels were then subjected to autoradiography overnight at $-80^{\circ} \mathrm{C}$.

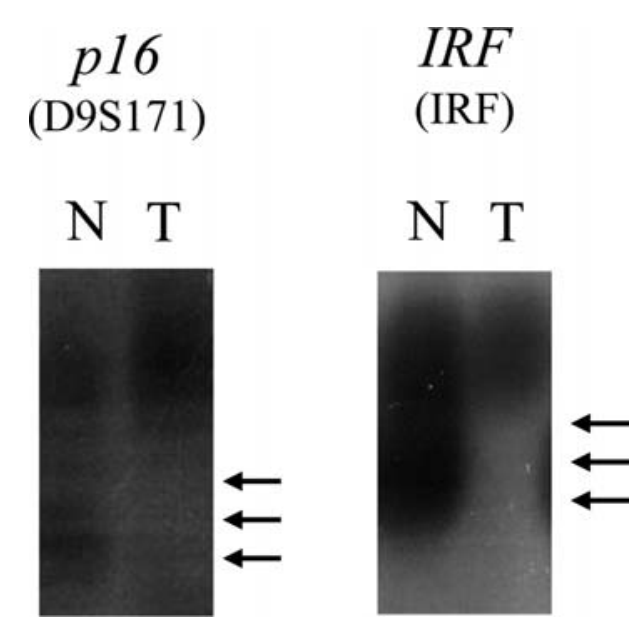

Figure 1. Representative examples of loss of heterozygosity analysis of p16 and $I R F . \mathrm{N}$, normal tissue; $\mathrm{T}$, tumor. Microsatellite loci are indicated in parentheses. Arrows indicate allelic loss.

\section{Results}

Genetic analyses of the multiple esophageal SCCs in the 30 patients are summarized in Table II. In microsatellite assay, if the lengths of microsatellite loci in alleles are identical, LOH status cannot be determined. Therefore, uninformative cases are inevitable. In LOH analysis of p53, 50 of the 67 tumors were informative, and $\mathrm{LOH}$ was detected in $16(32 \%)$ of the tumors (Fig. 1). For p16, 38 tumors were informative, and LOH was detected in $5(13 \%)$. For $I R F, 48$ tumors were informative, and $\mathrm{LOH}$ was detected in $5(10 \%)$. Mutation of 
p16 was detected in 4 of the $67(6 \%)$ tumors. Alteration of mtDNA was detected in 6 of the $67(9 \%)$ tumors and none of the tumors showed MSI-H. There were no significant relations between the mutations and clinicopathologic features (data not shown).

All 30 patients showed one or more gene alterations in one or more genetic loci. Discordant genetic patterns among multiple esophageal SCCs were observed in 28 of the 30 (93\%) patients. Only in the remaining $2(7 \%)$ patients was the genetic pattern among the tumors identical. The number of discordant genetic loci observed included 1 in 9 patients, 2 in 11 patients, 3 in 3 patients, 4 in 3 patients and 5 in 2 patients. The most discordant locus observed was that of TP53, occurring in 11 of $29(38 \%)$ informative cases, followed by D18S61, occurring in 11 of $30(37 \%)$ cases.

\section{Discussion}

In the present study, we showed that in most patients, the genetic pathways of multiple esophageal SCCs can differ within the same patient. To our knowledge, this is the first study to report such a finding.

The $p 53$ tumor suppressor gene has an open reading frame of 393 amino acids in length, and is located at the short arm of human chromosome 17 (14). Normal p53 functions in cell cycle regulation, in the maintenance of genomic stability and in controlled cell death (apoptosis). By preventing continued cell proliferation in the face of damaged DNA, p53 limits the likelihood of mutations becoming fixed, thereby acting as a 'guardian of the genome' (15). Frequent p53 mutations and p53 accumulation have been found in cases of esophageal SCC $(16,17)$. It has been reported that $p 53$ mutations occur as early events in $30-80 \%$ of esophageal SCCs. The results of the present study are consistent with those of previous investigations.

The tumor suppressor gene $p 16$ is located on chromosome 9 p21, a frequent site of $\mathrm{LOH}$ in many human malignancies. Giroux et al (18) reported that LOH of p16 was found in $14.7 \%$ of the SCCs investigated. In the present study, LOH of p16 was detected in 5 of 38 (13\%) informative cases. These results are consistent with those of Giroux et al. IRF, another tumor suppressor gene, is located on chromosome $5 q 31.1$ (19). LOH of this gene was reported to occur in 44$57 \%$ of esophageal tumors. In addition to alterations of these tumor suppressor genes, somatic mutations of mtDNA have previously been reported in esophageal SCCs (8). The reported frequencies varied from 9 to $34 \%$. Few esophageal SCCs show MSI-H.

In the present study, the frequency of $\mathrm{LOH}$ of $p 53$ and $p 16$, mutations of mtDNA, and MSI-H was consistent with those of previous studies, but the frequency of LOH of IRF was relatively lower than those of the previous reports. This may be due to the difference of genetic markers examined or to tumor stage differences.

Esophageal SCC is regarded as one of the representative examples of field carcinogenesis because the incidence of multiple occurrences of esophageal SCC is quite high in heavy smokers and heavy drinkers (20). Both tobacco smoking and alcohol drinking can induce genetic/epigenetic alterations in esophageal mucosal cells. Kammori et al (21) reported that telomere shortening in the esophageal epithelium is linked to carcinogenesis in esophageal SCC and field carcinogenesis in the esophagus.

It is of great interest to know whether multiple esophageal SCCs within a patient develop through identical or different genetic pathways. In the present study, discordant genetic patterns among individual lesions within a patient were observed in 28 of the 30 (93\%) patients, and this finding suggests that multiple esophageal SCCs in the same patient may develop via different genetic pathways. Discordant p53 mutations have been identified in esophageal SCC and preinvasive lesions from the same patient (22). These results can be explained by the theories of both field and multicentric carcinogenesis. Our results suggest further evidence of field or multicentric carcinogenesis of the human esophagus.

We previously reported the incidence of a speckled pattern of Lugol-voiding lesions (LVLs) of the background esophageal mucosa to be $83 \%$ in patients with metachronous multiple SCCs, $50 \%$ in those with synchronous SCCs and $27 \%$ in those with solitary SCC (1). LVLs are detectable in dysplastic lesions and in esophagitis, and the esophagus with a speckled pattern of LVLs may be at high risk for development of esophageal SCC. This finding may support the field carcinogenesis theory, although the genetic analysis in the present study did not show any definitive evidence of the field carcinogenesis.

Our results may explain the difficulty seen in treating esophageal SCCs with chemotherapy. Few esophageal tumors regress completely with chemotherapy. The genetic background of esophageal SCCs may vary considerably, whereas reagents used in chemotherapy generally target certain proteins or genes. Thus, target conditions may vary from tumor to tumor. In the future, the effect of chemotherapeutic agents may be predicted by detailed genetic analysis of the tumor.

In conclusion, our results suggest that genetic pathways of multiple esophageal SCCs may differ even within the same patient. Further investigation to clarify the genetic pathways of multiple esophageal SCCs may be needed. In addition, investigation into the genetic differences between solitary and multiple esophageal SCCs may also be necessary.

\section{References}

1. Urabe Y, Hiyama T, Tanaka S, Oka S, Yoshihara M, Arihiro K and Chayama K: Metachronous multiple esophageal squamous cell carcinomas and Lugol-voiding lesions after endoscopic mucosal resection. Endoscopy 41: 304-309, 2009.

2. Pesko P, Rakic S, Milicevic M, Bulajic P and Gerzic Z: Prevalence and clinicopathologic features of multiple squamous cell carcinoma of the esophagus. Cancer 73: 2687-2690, 1994.

3. Natsugoe S, Matsumoto M, Okumura H, et al: Multiple primary carcinomas with esophageal squamous cell cancer: clinicopathologic outcome. World J Surg 29: 46-49, 2005.

4. Nowell PC: The clonal evolution of tumor cell populations. Science 194: 23-28, 1976.

5. Strong MS, Incze J and Vaughan CW: Field cancerization in the aerodigestive tract - its etiology, manifestation, and significance. J Otolaryngol 13: 1-6, 1984.

6. Ito S, Ohga T, Saeki H, et al: p53 mutation profiling of multiple esophageal carcinoma using laser capture microdissection to demonstrate field carcinogenesis. Int J Cancer 113: 22-28, 2005.

7. Shima H, Hiyama T, Tanaka S, Yoshihara M, Arihiro K and Chayama K: Genetic progression and divergence in superficial esophageal squamous cell carcinoma by loss of heterozygosity analysis. Oncol Rep 16: 685-691, 2006. 
8. Kose K, Hiyama T, Tanaka S, Yoshihara M, Yasui W and Chayama K: Somatic mutations of mitochondrial DNA in digestive tract cancers. J Gastroenterol Hepatol 20: 1679-1684, 2005.

9. Hiyama T, Yoshihara M, Tanaka S and Chayama K: Genetic polymorphisms and esophageal cancer risk. Int J Cancer 121: 1643-1658, 2007.

10. Japanese Society for Esophageal Disease: Guidelines for Clinical and Pathological Studies on Carcinoma of the Esophagus, 10th edition. Kanehara, Tokyo, 2007.

11. Miyoshi E, Haruma K, Hiyama T, Tanaka S, Yoshihara M, Shimamoto F and Chayama K: Microsatellite instability is a genetic marker for the development of multiple gastric cancers. Int J Cancer 95: 350-353, 2001.

12. Hiyama T, Tanaka S, Yoshihara M, et al: Chromosomal and microsatellite instability in sporadic gastric cancer. J Gastroenterol Hepatol 19: 756-760, 2004

13. Hiyama T, Haruma K, Kitadai Y, et al: K-ras mutation in Helicobacter pylori-associated chronic gastritis in patients with and without gastric cancer. Int J Cancer 97: 562-566, 2002.

14. Lehrbach DM, Nita ME and Cecconello I: Molecular aspects of esophageal squamous cell carcinoma carcinogenesis. Arq Gastroenterol 40: 256-261, 2003.

15. Lane DP: Cancer: p53, guardian of the genome. Nature 358: 15-16, 1992.
16. Hollstein MC, Metcalf RA, Welsh JA, Montesano R and Harris CC: Frequent mutation of the p53 gene in human esophageal cancer. Proc Natl Acad Sci USA 87: 9058-9061, 1990.

17. Gao H, Wang LD, Zhou Q, Hong JY, Huang TY and Yang CS: p53 tumor suppressor gene mutation in early esophageal precancerous lesions and carcinoma among high-risk populations in Henan, China. Cancer Res 54: 4342-4346, 1994.

18. Giroux MA, Audrezet MP, Metges JP, et al: Infrequent p16/ CDKN2 alterations in squamous cell carcinoma of the esophagus. Eur J Gastroenterol Hepatol 14: 15-18, 2002.

19. Ogasawara S, Tamura G, Maesawa C, et al: Common deleted region on the long arm of chromosome 5 in esophageal carcinoma. Gastroenterology 110: 52-57, 1996.

20. Miyazaki M, Ohno S, Futatsugi M, Saeki H, Ohga T and Watanabe M: The relation of alcohol consumption and cigarette smoking to the multiple occurrence of esophageal dysplasia and squamous cell carcinoma. Surgery 131 (Suppl. 1): S7-S13, 2002.

21. Kammori M, Poon SS, Nakamura K, et al: Squamous cell carcinomas of the esophagus arise from a telomere-shortened epithelial field. Int J Mol Med 20: 793-799, 2007.

22. Benett WP, Hollstein MC, Metcalf RA, et al: p53 mutation and protein accumulation during multistep human esophageal carcinogenesis. Cancer Res 52: 6092-6097, 1992. 